\title{
Thermal radiation of gold clusters on microsecond time scales
}

\author{
K. Hansen,,${ }^{1,2,3, *}$ P. Ferrari, ${ }^{2}$ E. Janssens, ${ }^{2}$ and P. Lievens ${ }^{2, \dagger}$ \\ ${ }^{1}$ Tianjin International Center of Nanoparticles and Nanosystems, Tianjin University, Tianjin 300072, People's Republic of China \\ ${ }^{2}$ Laboratory of Solid State Physics and Magnetism, KU Leuven, 3001 Leuven, Belgium \\ ${ }^{3}$ Department of Physics, University of Gothenburg, 41296 Gothenburg, Sweden
}

(Received 1 October 2016; published 14 August 2017)

\begin{abstract}
Small positively charged gold clusters have been found to emit thermal radiation at a very high rate, with time constants ranging from one to $35 \mu \mathrm{s}$ for $\mathrm{Au}_{n}{ }^{+}(n=6-13,15)$. For sizes $n=14,16-20$ the radiation occurs on much longer time scales. Strong thermal suppression of the population of higher-lying states puts constraints on the possible energies of excited states that can contribute to the radiation. Taking that into account, an evaluation of the experimentally determined rate constants shows that the strong radiation originates from thermally excited low-lying electronic states hitherto not observed. The origin of these states is discussed and two possibilities are suggested: one is related to electron correlation and electron pairing, and the other results from thermal shape fluctuations.
\end{abstract}

DOI: 10.1103/PhysRevA.96.022511

\section{INTRODUCTION}

Bulk gold is the paradigm of noble metals. Nevertheless, gold clusters of sizes from a few to tens of atoms have shown a surprisingly strong reactivity [1-3] and have attracted considerable interest as catalysts. Experiments on, in particular, the catalytic properties of gold have mostly been performed on supported clusters, but the nature of the fundamental excitations and structure of these clusters are arguably better studied on free particles [4-6]. Benchmark experimental results are essential for a proper understanding of the structure of the clusters in both the ground and electronically excited states. This is of crucial importance to validate computational approaches, in particular for gold, for which relativistic effects are important quantum mechanically. Optical activity of gold clusters is potentially very important for the interpretation of experiments on abundances and stability and is obviously also intimately connected to plasmonic studies [7]. Potentially, thermal radiation and the concomitant size-dependent cooling of the clusters will have important consequences for mass production of size-selected clusters by the size-dependent enrichment of specific sizes, not only for gold clusters.

In the experiments reported here, the light-induced unimolecular decay of positively charged gold clusters was measured, allowing a determination of the radiative cooling of the clusters from thermally excited electronic states via the quenching effects on the unimolecular decay. Neutral $\mathrm{Au}$ clusters were ionized and fragmented by multiphoton absorption, and the metastable (delayed) fragmentation was quantified by means of mass spectrometry and compared with the hypothetic situation in which radiation cooling is absent $[8,9]$.

\section{EXPERIMENTAL PROCEDURE}

The experimental apparatus consists of a laser ablation cluster source and a time-of-flight mass spectrometer that

\footnotetext{
*klavshansen@tju.edu.cn

†peter.lievens@kuleuven.be
}

includes an excitation and acceleration region and a reflectron [10]. Solid-gold target material was ablated by pulsed 532-nm laser light, and the generated plasma was cooled by a heliumgas pulse with a backing pressure of 7 bars. After expansion and skimming of the gas-cluster mixture, neutral clusters entered the combined excitation, ionization, and extraction region, where they were photoexcited and ionized with a laser light pulse of $20 \mathrm{~mJ}$ at $355 \mathrm{~nm}$ or $140 \mathrm{~mJ}$ at $532 \mathrm{~nm}$. Charged clusters created directly in the source were rejected by a small static potential [9]. After ionization and a variable delay $\Delta t$, an extraction field was switched on. It corresponded to an ion energy of approximately $3.5 \mathrm{keV}$, depending slightly on the delay and therefore the precise position in the extraction region. The accelerated clusters had a $1.2-\mathrm{m}$ free flight before turning in the reflectron and were finally detected with a dualchannel plate detector $0.8 \mathrm{~m}$ downstream from the reflectron.

The net result of the combined excitation and ionization process is the creation of an ensemble of charged clusters of different sizes and wide internal energy distributions at the time of excitation and ionization. The clusters subsequently dissipated this excess energy in evaporative or radiative processes. The part of the evaporative decay cascade that occurred during free flight constitutes the measured signal. The energy distribution of each cluster size after excitation was very wide due to the nonconstant fluence and hence the number of absorbed photons across the laser beam profile. The effect of discrete photon energies was tested by changing the photon wavelengths and was found to be negligible (see below).

The unimolecular decay during the flight from the initial acceleration to the reflectron was monitored by detuning the potentials in the reflectron, a procedure that separates the flight times of those clusters that fragment in free flight, the metastable clusters, from those that do not [11]. A mass spectrum of gold clusters recorded under these conditions is shown in Fig. 1(a), where both prompt and metastable fragments are visible.

The metastable fraction $M_{n}$, defined as the intensity of metastable fragments normalized by the sum of the intensities of prompt and metastable clusters, is shown as a function of size in Fig. 1(b) at $\Delta t=0$ and for $\lambda=355$ and $532 \mathrm{~nm}$. 

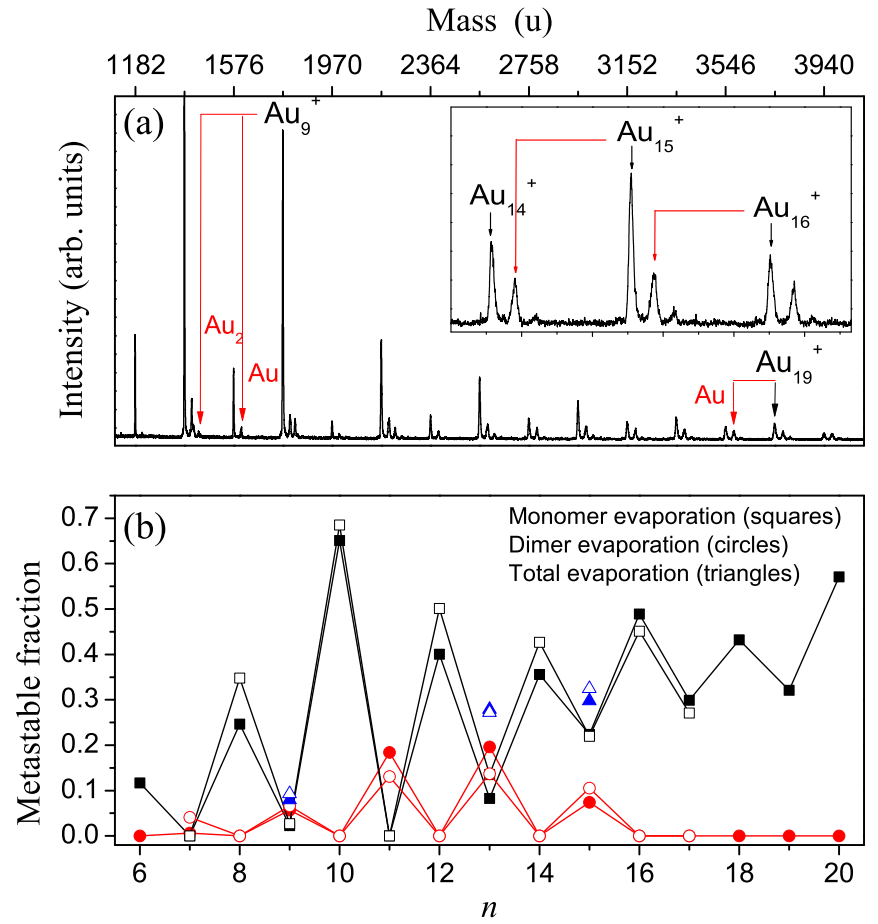

FIG. 1. (a) A mass spectrum showing the prompt and metastable fragments for $\Delta t=0$ after excitation with a wavelength of $355 \mathrm{~nm}$ and 20-mJ laser pulse energy. The metastable fragments of cluster sizes $n$ are visible as small peaks just to the right of the peak representing the prompt $n-1$ clusters. An occasional second side peak represents dimer loss from a $n+1$ cluster. (b) The metastable decay fraction vs cluster size for $\Delta t=0, \lambda=355 \mathrm{~nm}$ (solid symbols) and $\lambda=532 \mathrm{~nm}$ (open symbols). The general increase with cluster size correlates to the heat capacity and is well understood. The monomer (squares)-dimer (circles) evaporation branching ratios observed here are similar to those reported in [12]. Triangles show the sum of the monomer and dimer branches.

The wide distribution of excitation energies imparted onto the clusters by the exciting laser pulse causes the unimolecular decay to proceed via a power law in the absence of radiation, as demonstrated previously on a number of occasions (see, e.g., [13-20]). The quantization of the absorbed energy in the form of photons does not change this result, as a comparison of the metastable fractions for the two different photon energies shows [see Fig. 1(b)]. This is due to the widely different number of photons absorbed [21], which causes a cluster of a specific detected size to be produced from a range of different neutral precursors. A contributing factor is the energy smearing in the stochastic kinetic energy release in the unimolecular processes leading to the final size.

\section{RESULTS AND DATA ANALYSIS}

Thermal radiation is a nondestructive cooling process that competes with unimolecular reactions and suppresses the amount of metastable decay relative to the power law. The competition between evaporation and radiation for $\mathrm{Au}_{9}^{+}$is illustrated in Fig. 2, where the rate constants for the two processes are shown for $n=9$ (the details of calculations of such a value are given below). The salient point in Fig. 2 is

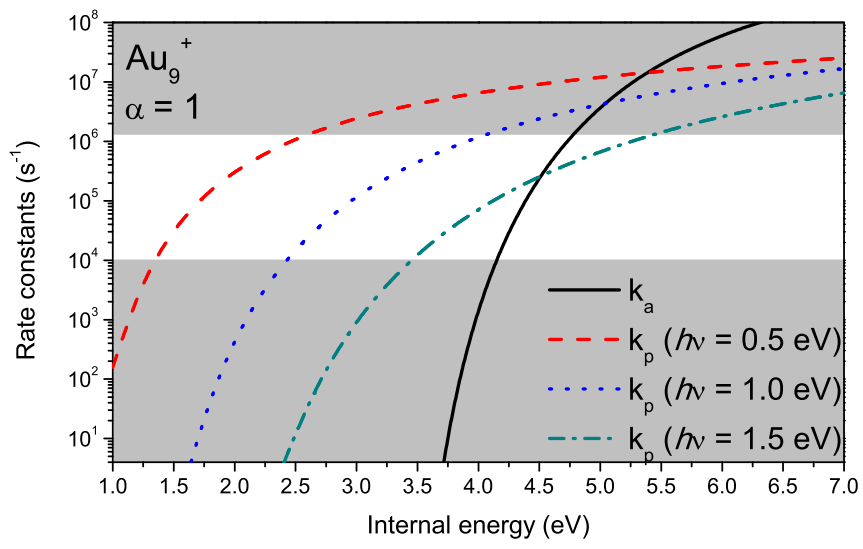

FIG. 2. Rate constants of fragmentation $k_{a}$ (solid black line) and radiation $k_{p}$ for $\mathrm{Au}_{9}^{+}$, with the energy of the emitting state being $h v_{0}=0.5 \mathrm{eV}$ (dashed red line), $h v_{0}=1.0 \mathrm{eV}$ (dotted blue line), and $h v_{0}=1.5 \mathrm{eV}$ (dash-dotted green line). The oscillator strength has been set to the value that exhausts the dipole sum rule $(\alpha=1)$. This is not realistic, but lower values are easily obtained by simply reducing the curves with a constant factor corresponding to the reduction in $\alpha$. The nonshaded area corresponds to the range of $k_{p}$ values that are accessible in these experiments.

that the photon emission rate constants are considerably less dependent on excitation energy than the evaporative decay constant. This means that even if the photon emission is an activated process, the energy dependence is still so weak that it can be considered approximately constant over the energy interval probed here.

Figure 3 shows the measured metastable fraction for three different cluster sizes as a function of $\ln \left(t_{2} / t_{1}\right)$, corresponding to an integrated power-law rate. The times $t_{1}$ and $t_{2}$ are the start and end of the period for which metastable decay can be measured with the help of the reflectron; that is, $t_{2}-t_{1}$ is the time of the free flight between the end of the initial acceleration and the entry into the reflectron. The start time $t_{1}$ for metastable evaporation is defined as the time interval between the laser pulse and the end of the acceleration, which for this purpose is reached when the cluster is at the potential halfway between the starting potential and ground potential.

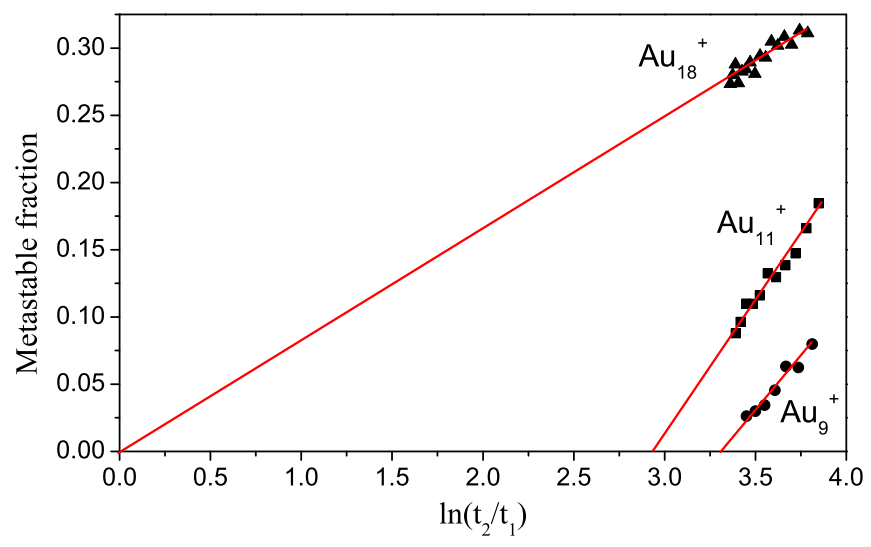

FIG. 3. The metastable fraction vs $\ln \left(t_{2} / t_{1}\right)$ for $\mathrm{Au}_{n}^{+}(n=9,11$, and 18). The negative intercept is clearly visible for $n=9$ and 11 . 
This time was determined in a separate experiment where the reflectron field was lowered in steps to measure the kinetic energy of the ions, determined as the potential where the ions penetrated the reflectron and were detected by a detector placed behind the high-potential side of the reflectron. The energy determination is equivalent to a determination of the position of ionization and, consequently, the calculation of $t_{1}$. An alternative control of the value used is given by the amount of metastable fragmentation of the nonradiating species. This was found to agree well with the amount predicted by the evaporative ensemble [22] for only monomer evaporation:

$$
M \approx \frac{C_{v} \ln \left(t_{2} / t_{1}\right)}{\ln \left(\omega t_{1}\right)^{2}}
$$

where $C_{v}$ is the heat capacity in units of $k_{B}$. This expression does not take size-to-size variations of binding energies into account, and the effects of dimer evaporation are not included either. The check is therefore only approximate. However, given that both values agree and that the sensitivity of the results reported below on $t_{1}$ was found to be low, this potentially critical parameter is therefore under good control.

The times $t_{1}$ and $t_{2}$ are varied in parallel by a few microseconds by changing the delay time $\Delta t$ of the pulsing on of the initial acceleration potentials; $t_{2}$ is on the order of a few tens of microseconds. Nonradiative decay will give a straight line with zero intercept in Fig. 3. Radiative cooling will make the ordinate intercept negative [9]. Note that this does not imply any model-dependent interpretation of the experimental data.

The radiative suppression of the power-law decay is exponential or near exponential. The precise time dependence is determined by the energy of the emitted photons relative to an energy scale determined by the (microcanonical) temperature, the heat capacity, and the evaporative activation energy $[9,23]$. For the energy of the photons expected to describe the behavior observed here, a single rate constant, $k_{p}$, gives the suppression directly (the continuous cooling approximation requires photon energies below $220 \mathrm{meV}$ for $n=15$; see [23]). The decay rate relevant here is then

$$
R \propto \int_{0}^{\infty} p(E) k_{a}(E) e^{-\left[k_{a}(E)+k_{p}(E)\right] t} d E
$$

where $k_{a}(E)$ is the energy-dependent rate constant for atomic (or dimer, where relevant) evaporation, $k_{p}(E)$ is the photon emission rate constant, and $p(E)$ is the density of excitation energies. Irrespective of the precise magnitude of the emitted photon energy, $k_{p}$ can be set constant because the variation of that function is much slower than the other factors in the integrand, as mentioned (see also, e.g., [24,25]). This fact follows from the rigorous limits on the frequency factor for photon emission rate constants imposed by the dipole oscillator sum rules, given explicitly in Eq. (5). This simplification yields the unimolecular decay rate:

$$
R \propto \frac{e^{-k_{p} t}}{t}
$$

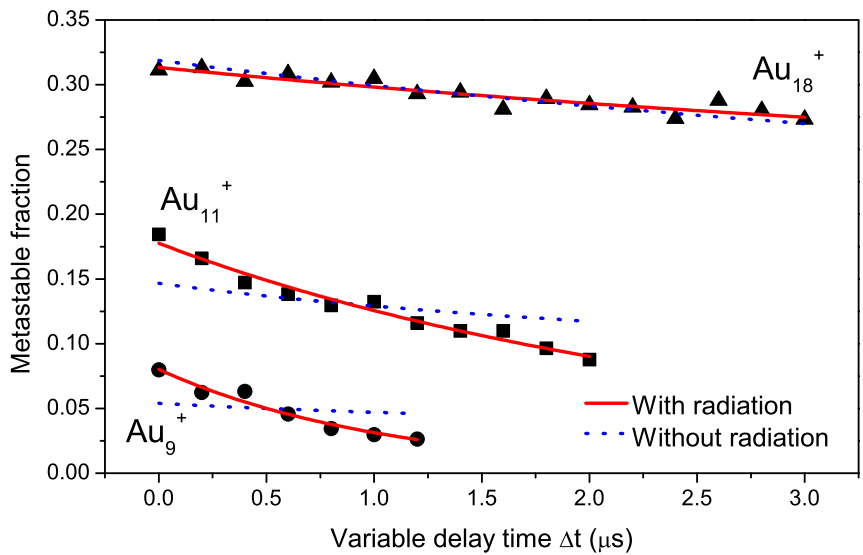

FIG. 4. Fits of the radiative cooling time constant with Eq. (4) for $\mathrm{Au}_{n}^{+}(n=9,11$, and 18). Symbols are the data, the blue dotted line is the nonradiative fit [i.e., setting $k_{p}=0$ in Eq. (4)], and the red solid line is the fit without any restrictions on $k_{p}$.

Integrating this expression between $t_{1}$ and $t_{2}$ gives the observed metastable fraction:

$$
M_{n} \propto \int_{t_{1}}^{t_{2}} R d t \propto \int_{k_{p} t_{1}}^{k_{p} t_{2}} x^{-1} e^{-x} d x .
$$

The radiative rate constants are extracted from fits of the data with this equation. Fits for three cluster sizes are shown in Fig. 4. The radiationless fit curves are not only a poorer fit for $n=9,11$; the absolute measured values of the amount of metastable fragmentation are also significantly lower than the expected non-radiative values.

The fitted values of $k_{p}$ are shown in Fig. 5(a). The values are highly size dependent, with strong odd-even alternations
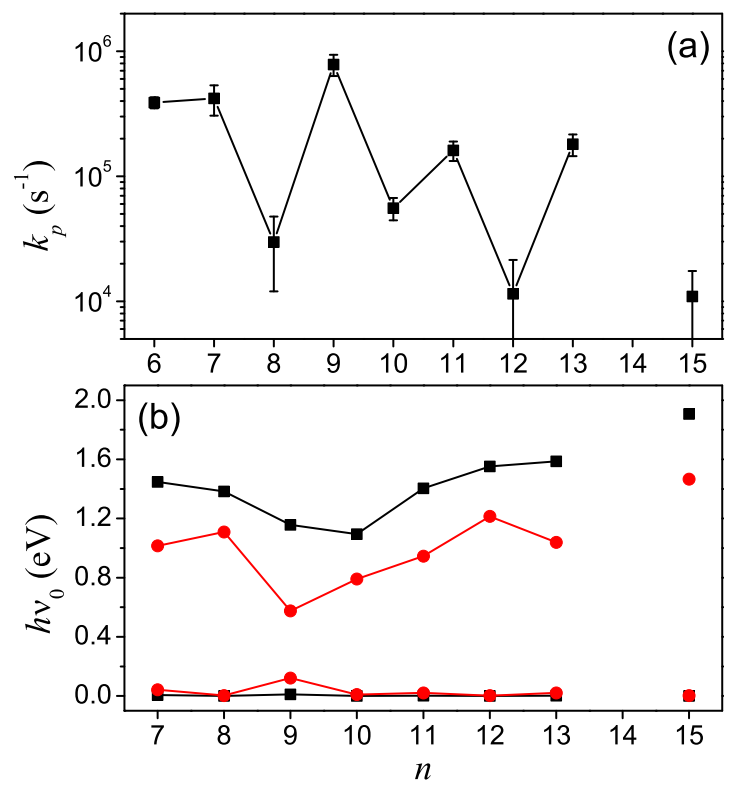

FIG. 5. (a) The fitted values of $k_{p}$. The uncertainties are $1 \sigma$ values. The values for $k_{p} n=14,16-20$ are consistent with zero. (b) Upper and lower limits of possible state energies for oscillator strengths corresponding to one electron oscillator strength (red circles) or $n-1$ valence electrons (black squares). 
similar to the ones seen in abundance spectra [4] and, with smaller amplitudes, in binding energies [26]. Several of these measured radiation times are very short. The highest value of $k_{p}$ at $n=9$, for example, corresponds to a radiative time constant of $1.3 \mu \mathrm{s}$. They cannot be explained by the radiation of vibrational transitions, which is typically three or more orders of magnitude slower. The radiation must therefore involve electronic transitions. Attempts to explain the emission from the low-energy tail of the surface plasmon, such as for fullerenes [27], did not give sufficiently high rates to explain the present observation with realistic parameters for the surface plasmon resonance for gold. Neither does spectroscopic evidence support this suggestion [28-30]. These studies probe the visible and ultraviolet regimes and do not show the very high oscillator strength of a surface plasmon for these sizes, in contrast to the spectra of larger, nanometer-sized Au particles [31] and the fullerenes.

The remaining possibility is radiation from thermally populated low-lying excited electronic states, corresponding to recurrent fluorescence, also termed Poincaré fluorescence. This type of radiation was proposed a long time ago [32,33] but was only recently detected for several excited carbon species $[19,25,34,35]$ and very recently also confirmed by direct detection of emitted photons for $\mathrm{C}_{6}{ }^{-}$[36].

\section{DISCUSSION}

Recurrent fluorescence makes the photon emission an activated process with a rate constant given essentially by the product of the Einstein $A$ coefficient of the excited state and the thermal population. Dipole oscillator sum rules combined with the experimentally determined $k_{p}$ values impose severe constraints on the possible energies of excited states that can contribute to the radiation. The photon emission rate constant from a single excited state with energy $h v_{0}$ reads [37]

$$
k_{p}=7.421 \times 10^{-22} \mathrm{~Hz}^{-1} f v_{0}^{2} \frac{\frac{\rho\left(E-h v_{0}\right)}{\rho(E)}}{1-\frac{\rho\left(E-2 h \nu_{0}\right)}{\rho\left(E-h \nu_{0}\right)}},
$$

where $\rho(E)$ is the cluster level density at excitation energy $E$ and $f(\leqslant n-1)$ is the oscillator strength limited by the Thomas-Reiche-Kuhn dipole transition sum rule. By detailed balance this sum rule for the absorption cross section also gives a rigorous upper limit for the emission rate constant. This, in turn, combined with the suppressing effect of the ratio of level densities, limits the possible values of $h v_{0}$.

We find this limit by expressing the ratios of level densities as a Boltzmann factor, using the microcanonical temperature. This procedure cancels most of the uncertainties associated with the unknown thermal properties of the gold clusters. With $i=0,1$ the ratio of level densities in Eq. (5) can be expressed as

$$
\frac{\rho[E-(i+1) h v]}{\rho(E-i h v)} \approx \exp \left(-\frac{h v}{T(E)-\frac{2 i+1}{2 C_{v}} h v}\right),
$$

where $T(E)$ is the microcanonical temperature of the cluster before emission. A similar expression enters the unimolecular rate constant $k_{a}$, with $i=0$ and the substitution of $h v$ by the evaporative activation energy $D_{n}$. As the unimolecular rate constant has the strongest energy dependence, this determines

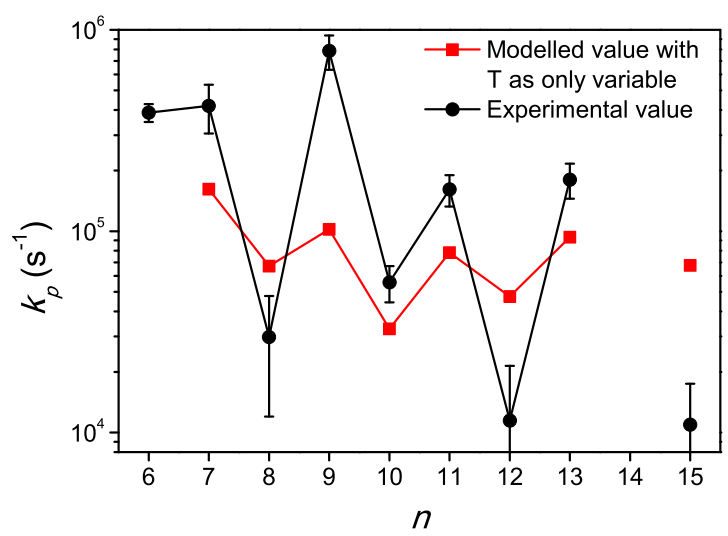

FIG. 6. Radiative cooling rates for identical parameters, except the cluster temperature (red squares) compared with fitted values (black circles).

the temperature, which is then found by setting $k_{a}=1 / t_{2}$ to

$$
T_{n}=D_{n}\left(\frac{1}{\ln \left(\omega_{a} t_{2}\right)}+\frac{1}{2 C_{v}}\right),
$$

where $\omega_{a}$ is the frequency factor for atomic evaporation. Both $\omega_{a}$ and $D$ 's are known experimentally for cationic clusters with $n=7-27$ [26]. The values are $\omega_{a}=2.4 \times 10^{15} \mathrm{~s}^{-1}$, common for all sizes, and the size-specific dissociation energies range from $2.4 \mathrm{eV}$ for $n=8$ to $3.84 \mathrm{eV}$ for $n=21$ [26]. This determines the $T$ 's that enter Eq. (6). The numerical value of the preexponential factor in Eq. (5) is $4.38 \times 10^{7} \mathrm{~s}^{-1} f$ for a $1 \mathrm{eV}$ photon. As the temperature given by Eq. (7) has an odd-even variation, the strong odd-even variations in the radiation constants could potentially be a simple consequence of this. To this end we calculated how much thermal radiation is emitted by different cluster sizes purely on the basis of different dissociation energies. Herein, experimental dissociation energies are taken from Ref. [26], and the microcanonical temperature $T$ of the metastable clusters is calculated with Eq. (7). Assuming a common frequency factor of $10^{6} \mathrm{~s}^{-1}$ for radiation and that all cluster sizes radiate from an electronic transition at $0.5 \mathrm{eV}$, the rate of radiation is calculated as shown with red squares in Fig. 6. These resulting $k_{p}$ values should be compared with photon emission rate constants extracted from the fits of the data (black dots). From the results in Fig. 6 we conclude that at least either the excited-state energies or the oscillator strengths must possess an odd-even variation.

The experimental results and Eq. (5) determine the possible ranges for $h v_{0}$. Figure 5(b) shows the upper and lower limits for the energy of the excited state $h v_{0}$ for the two values $f=n-1$, corresponding to a transition which absorbs all valence electron dipole oscillator strength, and $f=1$, a more realistic oscillator strength corresponding to a single electron. Due to the strong dependence of the population on $h v_{0}$, the difference between the two is not large. The lower energy limits are due to the phase-space factor of the photon, which varies as $v^{2}$.

Figure 7 summarizes the relation between $f$ and the state energy for $n=7-13,15$, imposed by the measured radiation time constants assuming the radiation appears from a single state. At high transition energy, the required oscillator strength 


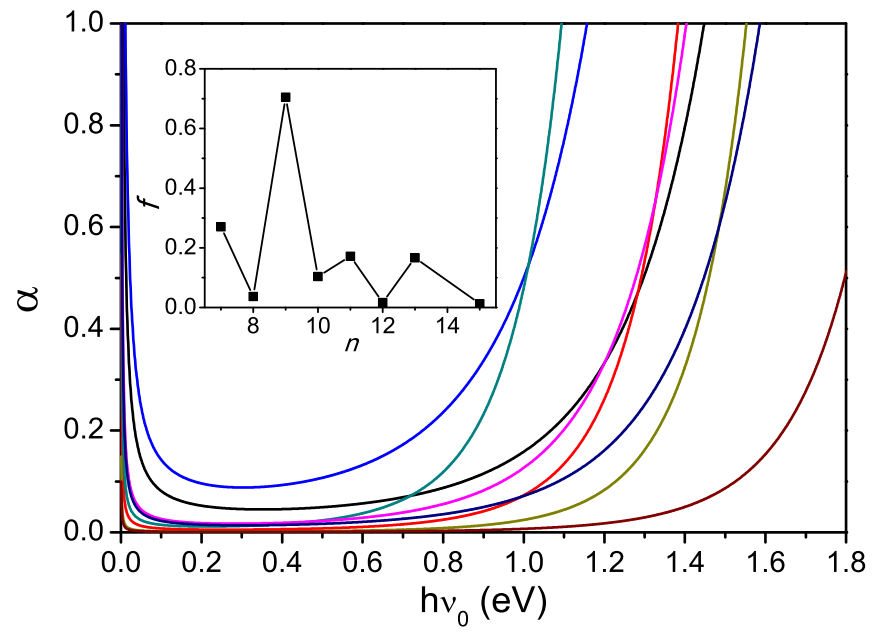

FIG. 7. The relation between the oscillator strength $\alpha \equiv f /(n-$ 1) and the state energy. The lines show, at $\alpha=1$ and from right to left, the values for $n=15,13,7,12,11,9,8,10$. The inset shows the smallest possible oscillator strengths consistent with the observed radiation.

grows almost exponentially with the photon energy. At lower energy it diverges due to the vanishing phase space of the photons. Combined, these effects define a minimum oscillator strength required for consistency with the experimental data. They are given in the inset as $f$ vs $n$.

States with this low excitation energy and with sufficient oscillator strength to compensate for the Boltzmann-factor suppression are hitherto unobserved for gold clusters. Experimentally, this energy range is beyond the reach of most action spectroscopy experimental approaches. The widest region studied experimentally covers from 1.5 to $6 \mathrm{eV}$ and reports an oscillator strength that decreases with size for the range $n=$ $1-5,7-9$ [30]. The quoted number is an oscillator strength of $15 \%$ of the total with, however, an unknown and possibly large correction assigned to matrix effects. Even after application of a rather liberal uncertainty, there should still be sufficient oscillator strength available for the proposed new transitions. A few theoretical studies on gold clusters have predicted low-energy states $[38,39]$. These studies are restricted to the smallest sizes (dimer, trimer, and tetramer) and use completeactive-space multiconfiguration self-consistent field theory with multireference configuration-interaction calculations to include electron correlations at a relatively high level.

Another theory that has predicted low-lying excited states was proposed many years ago by Iachello and coworkers
[40,41] and also involves electron correlations, in this case through excitations of pairs of electrons, analogous to descriptions of proton or neutron pairs in atomic nuclei [42]. The existence of such modes was invoked to explain broadening of plasmonic excitations in sodium clusters as a manifestation of electron correlation [40]. In many-fermion systems like clusters and atomic nuclei an interesting common aspect is that fermions couple and form pairs that are responsible for collective behavior, involving one or more pairs of nucleons (for atomic nuclei) or electrons (for clusters, molecules, and superconductors). There have been several studies invoking BCS mechanisms to predict superconducting behavior in (networks of) atomic clusters, so far with limited experimental evidence [43]. But manifestations that may relate to electron pairing, e.g., odd-even effects in experiments, are ubiquitous.

Yet another suggestion is that the transitions are intershell or subshell transitions. In the shapes defined by the vibrational and electronic ground states, these transitions may be beyond the reach of thermal excitations. Thermal shape fluctuations may, however, change this picture. A deformation will reduce the energy of some states and increase it for others. An explicit calculation of the effect for $\mathrm{Al}_{13}$ - in [44], based on a spherical box potential of finite depth, shows that level crossing can, indeed, be expected after photoexcitation. This mechanism potentially explains the observation of highintensity electronic radiation in other metal clusters [8,9], as well as the often very broad spectra measured when the photons are observed directly [45], although the broad cluster size distribution in that study will undoubtedly also play a role.

\section{SUMMARY}

We have found that small gold clusters radiate thermally with a very short time constant and very strong size dependence. The origin of the strong radiation has been traced to low-lying, hitherto unidentified, electronic states. These findings can be expected to be relevant for other cluster systems. Also, the high rate of radiative decay is an important factor in the chemical equilibration processes of clusters, and radiative cooling is therefore also potentially important for bulk production of size-selected clusters.

\section{ACKNOWLEDGMENTS}

This work is supported by the Research FoundationFlanders (FWO) and by the KU Leuven Research Council (GOA/14/ 007). P.F. acknowledges CONICyT for the Becas Chile scholarship.
[1] M. Haruta, Catal. Today 36, 153 (1997).

[2] H. Häkkinen, S. Abbet, A. Sanchez, U. Heiz, and U. Landman, Angew. Chem., Int. Ed. 42, 1297 (2003).

[3] H. Häkkinen, Nat. Chem. 4, 443 (2012).

[4] I. Katakuse, T. Ichihara, Y. Fujita, T. Matsuo, T. Sakurai, and H. Matsuda, Int. J. Mass Spectrom. Ion Processes 67, 229 (1985).

[5] I. Rabin, C. Jackschath, and W. Schulze, Z. Phys. D 19, 153 (1991).
[6] M. Vogel, K. Hansen, A. Herlert, and L. Schweikhard, Phys. Rev. Lett. 87, 013401 (2001).

[7] C. Sönnichsen, T. Franzl, T. Wilk, G. von Plessen, and J. Feldmann, New J. Phys. 4, 93 (2002).

[8] K. Hansen, Y. Li, V. Kaydashev, and E. Janssens, J. Chem. Phys. 141, 024302 (2014).

[9] P. Ferrari, E. Janssens, P. Lievens, and K. Hansen, J. Chem. Phys. 143, 224313 (2015). 
[10] W. Bouwen, P. Thoen, F. Vanhoutte, S. Bouckaert, F. Despa, H. Weidele, R. E. Silverans, and P. Lievens, Rev. Sci. Instrum. 71, 54 (2000).

[11] H. Kühlewind, H. J. Neusser, and E. W. Schlag, Int. J. Mass Spectrom. Ion Processes 51, 255 (1983).

[12] M. Vogel, A. Herlert, and L. Schweikhard, J. Am. Soc. Mass Spectrom. 14, 614 (2003).

[13] K. Hansen and E. Campbell, J. Chem. Phys. 104, 5012 (1996).

[14] J. U. Andersen, C. Brink, P. Hvelplund, M. O. Larsson, B. Bech Nielsen, and H. Shen, Phys. Rev. Lett. 77, 3991 (1996).

[15] K. Hansen, J. U. Andersen, P. Hvelplund, S. P. Møller, U. V. Pedersen, and V. V. Petrunin, Phys. Rev. Lett. 87, 123401 (2001).

[16] J. U. Andersen, H. Cederquist, J. S. Forster, B. A. Huber, P. Hvelplund, J. Jensen, B. Liu, B. Manil, L. Maunoury, S. Brøndsted Nielsen et al., Eur. Phys. J. D 25, 139 (2003).

[17] L. Andersen, O. Heber, and D. Zajfman, J. Phys. B 37, R57 (2004).

[18] M. W. Froese, K. Blaum, F. Fellenberger, M. Grieser, M. Lange, F. Laux, S. Menk, D. A. Orlov, R. Repnow, T. Sieber et al., Phys. Rev. A 83, 023202 (2011).

[19] S. Martin, J. Bernard, R. Brédy, B. Concina, C. Joblin, M. Ji, C. Ortega, and L. Chen, Phys. Rev. Lett. 110, 063003 (2013).

[20] K. Hansen, M. H. Stockett, M. Kaminska, R. F. Nascimento, E. K. Anderson, M. Gatchell, K. C. Chartkunchand, G. Eklund, H. Zettergren, H. T. Schmidt et al., Phys. Rev. A 95, 022511 (2017).

[21] K. Mehlig, K. Hansen, M. Hedén, A. Lassesson, A. V. Bulgakov, and E. E. B. Campbell, J. Chem. Phys. 120, 4281 (2004).

[22] K. Hansen, Statistical Physics of Nanoparticles in the Gas Phase, Springer Series on Atomic, Optical, and Plasma Physics Vol. 73 (Springer, Dordrecht, 2013).

[23] K. Najafian, M. S. Pettersson, B. Dynefors, H. Shiromaru, J. Matsumoto, H. Tanuma, T. Furukawa, T. Azuma, and K. Hansen, J. Chem. Phys. 140, 104311 (2014).

[24] J. Andersen, C. Gottrup, K. Hansen, P. Hvelplund, and M. Larsson, Eur. Phys. J. D 17, 189 (2001).

[25] N. Kono, T. Furukawa, H. Tanuma, J. Matsumoto, H. Shiromaru, T. Azuma, K. Najafian, M. S. Pettersson, B. Dynefors, and K. Hansen, Phys. Chem. Chem. Phys 17, 24732 (2015).
[26] K. Hansen, A. Herlert, L. Schweikhard, and M. Vogel, Phys. Rev. A 73, 063202 (2006).

[27] J. U. Andersen and E. Bonderup, Eur. Phys. J. D 11, 413 (2000).

[28] B. A. Collings, K. Athanassenas, D. Lacombe, D. M. Rayner, and P. A. Hackett, J. Chem. Phys. 101, 3506 (1994).

[29] S. Gilb, K. Jacobsen, D. Schooss, F. Furche, R. Ahlrichs, and M. M. Kappes, J. Chem. Phys. 121, 4619 (2004).

[30] S. Lecoultre, A. Rydlo, C. Félix, J. Buttet, S. Gilb, and W. Harbich, J. Chem. Phys. 134, 074302 (2011).

[31] E. Cottancin, G. Celep, J. Lermé, M. Pellarin, J. R. Huntzinger, J. L. Vialle, and M. Broyer, Theor. Chem. Acc. 116, 514 (2006).

[32] A. Nitzan and J. Jortner, J. Chem. Phys. 71, 3524 (1979).

[33] A. Léger, P. Boissel, and L. d'Hendecourt, Phys. Rev. Lett. 60, 921 (1988).

[34] G. Ito, T. Furukawa, H. Tanuma, J. Matsumoto, H. Shiromaru, T. Majima, M. Goto, T. Azuma, and K. Hansen, Phys. Rev. Lett. 112, 183001 (2014).

[35] V. Chandrasekaran, B. Kafle, A. Prabhakaran, O. Heber, M. Rappaport, H. Rubinstein, D. Schwalm, Y. Toker, and D. Zajfman, J. Phys. Chem. Lett. 5, 4078 (2014).

[36] Y. Ebara, T. Furukawa, J. Matsumoto, H. Tanuma, T. Azuma, H. Shiromaru, and K. Hansen, Phys. Rev. Lett. 117, 133004 (2016).

[37] J. U. Andersen, E. Bonderup, and K. Hansen, J. Phys. B: At. Mol. Opt. Phys. 35, R1 (2002).

[38] K. Balasubramanian, J. Mol. Struct.: THEOCHEM 202, 291 (1989).

[39] K. Balasubramanian and K. Das, Chem. Phys. Lett. 186, 577 (1991).

[40] F. Iachello, E. Lipparini, and A. Ventura, Phys. Rev B 45, 4431 (1992).

[41] F. Iachello, Nucl. Phys. A 570, 145 (1994).

[42] R. A. Broglia and V. Zelevinsky, Fifty Years of Nuclear BCS: Pairing in Finite Systems (World Scientific, Singapore, 2013).

[43] A. Halder, A. Liang, and V. V. Kresin, Nano Lett. 15, 1410 (2015).

[44] V. V. Kresin and Y. N. Ovchinnikov, Phys. Rev. B 73, 115412 (2006).

[45] U. Frenzel, U. Hammer, H. Westje, and D. Kreisle, Z. Phys. D 40, 108 (1997). 